\title{
ON THE DIFFRACTION OF A CYLINDRICAL PULSE BY A HALF-PLANE*
}

\author{
BY \\ G. E. BARR \\ Sandia Corporation, Albuquerque, New Mexico
}

The diffraction of electromagnetic or acoustic pulses by a wedge (and by its special case, a half plane) has been treated in a number of recent contributions. The majority of these treatments is based on a direct attack on the wave equation of arbitrary time dependency, with the following cases being treated: Normal incidence of a plane pulse on a half plane [6], [7] ${ }^{* *}$; two dimensional pulse incident on a half plane [7]; plane unit step function pulse incident on a wedge [13], [15], [16]; arbitrary pulse incident on a wedge [12]. For most of the rest, the approach centers on integral transform methods. The case of a spherical pulse (unit step function) incident on a half plane [22], uses the Fourier Transform method, while the case of a cylindrical "Dirac" pulse incident on a half plane [21] uses the Kontorovich-Lebedev transform method [14].

We wish to solve the wave equation for the potential for the case of a cylindrical "Dirac" pulse incident on a perfectly reflecting half plane [21]. The procedure used here, essentially similar to Cagniard's method [1], [3], [4], gives the solution to this problem not only in elementary form but in terms of functions of algebraic character.

The procedure is as follows: First one determines the Green's functions $G_{1}^{\prime}$ and $G_{2}^{\prime}$ for the so-called "modified" Helmholtz equation $\Delta u-\gamma^{2} u=0$, itself obtained from the Helmholtz equation $\Delta u+k^{2} u=0$ upon putting $k=-i \gamma$. (The velocity of propagation is set equal to one so $k=\omega$.) The Green's functions $G_{1}^{\prime}$ and $G_{2}^{\prime}$ are then cast in the form of Laplace transform integral $\int_{0}^{\infty} F(t) e^{-r t} d t$. Their inverse Laplace transform with respect to $\gamma$ as parameter is the "Dirac" pulse solution for the wave equation, that is, the Green's functions for the wave Eq. [1], [3], [4], and [9]. From the solution for the "Dirac" pulse the effect of an arbitrary pulse excitation $g(t)$ can be synthesized. Several special cases for $g(t)$ are examined.

I. Green's Functions. The Green's functions $G_{1}^{\prime}$ and $G_{2}^{\prime}$ for the wedge for the Dirichlet and Neumann problems, respectively for the modified Helmholtz equation are well known to be:

$$
\left.\begin{array}{l}
G_{1}^{\prime} \\
G_{2}^{\prime}
\end{array}\right\}=-\frac{1}{2 \alpha} \sum_{n=0}^{\infty} \epsilon_{n}\left\{\cos \frac{n \pi}{\alpha}\left(\phi-\phi^{\prime}\right) \mp \cos \frac{n \pi}{\alpha}\left(\phi+\phi^{\prime}\right)\right\} I_{n \pi / \alpha}(\gamma \rho) K_{n \pi / \alpha}\left(\gamma \rho^{\prime}\right)
$$

for $\rho^{\prime}>\rho$ and the same formula for $\rho>\rho^{\prime}$ save $\rho$ and $\rho^{\prime}$ are interchanged, where $Q\left(\rho^{\prime}, \phi^{\prime}\right)$ is the location of the line source and $P(\rho, \phi)$ is the location of the observer [18]. If we open the wedge so $\alpha$, the angle of opening, is $2 \pi$ we have the Green's functions for the half plane, namely:

${ }^{*}$ Received August 23, 1965; revised manuscript received July 5, 1966. This paper is part of a thesis submitted in partial fulfillment of the requirements for the degree of Doctor of Philosophy in Physics at Oregon State University.

**Numbers in square brackets refer to the bibliography at the end of this paper. 


$$
\left.\begin{array}{l}
G_{1}^{\prime} \\
G_{2}^{\prime}
\end{array}\right\}=-\frac{1}{4 \pi} \sum_{n=0}^{\infty} \epsilon_{n}\left\{\cos \frac{n}{2}\left(\phi-\phi^{\prime}\right) \mp \cos \frac{n}{2}\left(\phi+\phi^{\prime}\right)\right\} I_{n / 2}(\gamma \rho) K_{n / 2}\left(\gamma \rho^{\prime}\right),
$$

for $\rho<\rho^{\prime}$ and the same formula with $\rho$ and $\rho^{\prime}$ interchanged for $\rho>\rho^{\prime}$.

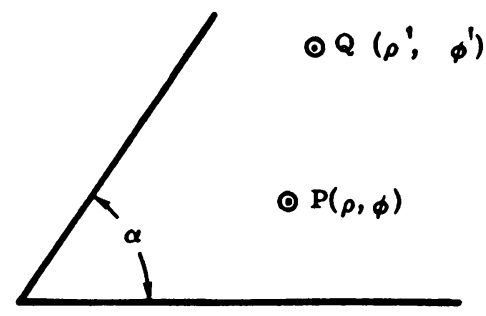

FIG. 1. Wedge of opening angle $\alpha$ showing the location of the line source $Q\left(\rho^{\prime}, \phi^{\prime}\right)$ and the observer $P(\rho, \phi)$.

II. Expression of $G_{1}^{\prime}$ and $G_{2}^{\prime}$ as a Laplace transform. Our goal is to express $G_{1}^{\prime}$ and $G_{2}^{\prime}$ as a Laplace Transform integral with $\gamma$ as transformation parameter, i.e., $G_{1}^{\prime}$ and $G_{2}^{\prime}$ should be of the form:

$$
\left.\begin{array}{l}
G_{1}^{\prime} \\
G_{2}^{\prime}
\end{array}\right\}=\int_{0}^{\infty} F(t) e^{-\gamma t} d t
$$

where $F(t)$ contains, besides the integration variable $t$, the properties $\alpha, \phi, \phi^{\prime}, \rho, \dot{\rho}^{\prime}$ as parameters, but is independent of $\gamma$. Then the inverse Laplace Transform of $G_{1}^{\prime}$ and $G_{2}^{\prime}$, $\mathcal{L}^{-1}\left\{G_{1,2}^{\prime}\right\}$ is just $F(t)$. For this purpose it is desirable to represent the term

$$
K_{n / 2}\left(\gamma \rho^{\prime}\right) I_{n / 2}(\gamma \rho)
$$

occurring in $G_{1}^{\prime}$ and $G_{2}^{\prime}$ in such a form. We use the representation

$$
\begin{aligned}
I_{\nu}(b s) K_{\nu}(a s)=\frac{1}{2}(a b)^{-1 / 2}\left\{\int_{a-b}^{a+b} P_{\nu-1 / 2}\right. & \left(\frac{a^{2}+b^{2}-t^{2}}{2 a b}\right) e^{-s t} d t \\
& \left.+\frac{2}{\pi} \cos (\pi \nu) \int_{a+b}^{\infty} Q_{\nu-1 / 2}\left(\frac{t^{2}-a^{2}-b^{2}}{2 a b}\right) e^{-a t} d t\right\}
\end{aligned}
$$

for $a>b, \operatorname{Re} s>0 . P$ and $Q$ are Legendre functions of the first and second kinds respectively. This representation appears to be new and is derived in the appendix. Now, replace $I_{n \pi / \alpha}(\gamma \rho) K_{n \pi / \alpha}\left(\gamma \rho^{\prime}\right)$ in (1) by (4) while putting $\nu=n \pi / \alpha, s=\gamma, b=\rho, a=\rho^{\prime}$. Upon interchanging the order of summation and integration which is permissible because of the absolute convergence of both sum (1) and integral (4) we encounter terms of the form

$$
\sum_{n=0}^{\infty} \epsilon_{n} P_{n \pi / \alpha-1 / 2}\left(\frac{\rho^{2}+\rho^{\prime 2}-t^{2}}{2 \rho \rho^{\prime}}\right) \cos \left(\frac{n \pi}{\alpha} v\right)
$$

and

$$
\sum_{n=0}^{\infty} \epsilon_{n} \cos \left(\frac{n \pi}{\alpha}\right) Q_{n \pi / \alpha-1 / 2}\left(\frac{t^{2}-\rho^{2}-\rho^{\prime 2}}{2 \rho \rho^{\prime}}\right) \cos \left(\frac{n \pi}{\alpha} v\right)
$$

where $v=\phi \mp \phi^{\prime}$.

Series (5) can be summed for an arbitrary opening angle $\alpha$ for the wedge, while presently (6) can be summed only for $\alpha=2 \pi$, namely the half plane. Then the Green's 
functions $G_{1}^{\prime}$ and $G_{2}^{\prime}$ for the modified Helmholtz equation can be given in the desired form of a Laplace Transform integral (3). The summations of these series are presented in the appendix. Henceforth we will consider the case $\alpha=2 \pi$, the half plane, exclusively.

Then, by (2), (4)

$$
\begin{aligned}
\sum_{n=0}^{\infty} \epsilon_{n} I_{n / 2}(\gamma \rho) K_{n / 2}\left(\gamma \rho^{\prime}\right) \cos \left(\frac{n v}{2}\right) & \\
= & \frac{1}{2}\left(\rho \rho^{\prime}\right)^{1 / 2}\left\{\int_{\rho^{\prime}-\rho}^{\rho^{\prime+\rho}}\left[\sum_{n=0}^{\infty} \epsilon_{n} \cos \left(\frac{n v}{2}\right) P_{n / 2-1 / 2}\left(\frac{\rho^{2}+{\rho^{\prime}}^{2}-t^{2}}{2 \rho \rho^{\prime}}\right)\right] e^{-\gamma t} d t\right. \\
& \left.+\frac{2}{\pi} \int_{\rho^{\prime}+\rho}^{\infty}\left[\sum_{n=0}^{\infty}(-1)^{n} \epsilon_{n} \cos (n v) Q_{n-1 / 2}\left(\frac{t^{2}-\rho^{2}-{\rho^{\prime 2}}^{2}}{2 \rho \rho^{\prime}}\right)\right] e^{-\gamma t} d t\right\}
\end{aligned}
$$

The sums under the integral signs are given in the appendix (2), (3).

At first we consider the second term on the right hand side of $(7)$ and, from the appendix (3), obtain

$$
\begin{aligned}
\int_{\rho+\rho^{\prime}}^{\infty}\left[\sum_{n=0}^{\infty}(-1)^{n} \epsilon_{n} \cos (n v) Q_{n}-\frac{1}{2}\left(\frac{t^{2}-\rho^{2}-{\rho^{\prime}}^{2}}{2 \rho \rho^{\prime}}\right)\right] e^{-\gamma t} d t \\
=\pi\left(\rho \rho^{\prime}\right)^{-1 / 2} \int_{\rho+\rho^{\prime}}^{\infty}\left[t^{2}-\left(\rho^{2}+{\rho^{\prime}}^{2}-2 \rho \rho^{\prime} \cos v\right)\right]^{-1 / 2} e^{-\gamma t} d t .
\end{aligned}
$$

Since $z=\left(t^{2}-\rho^{2}-{\rho^{\prime}}^{2}\right) / 2 \rho \rho^{\prime} \geq 1$ along the whole interval of integration $t=\rho+\rho^{\prime}$ to $t=\infty$, the condition for the validity of the sum (3), appendix is met. For the first term on the right hand side of (7), we have at first, by (2), appendix,

$$
\sum_{n=0}^{\infty} \epsilon_{n} \cos \left(\frac{n v}{2}\right) P_{1 / 2+n / 2}\left(\frac{\rho^{2}+\rho^{\prime 2}-t^{2}}{2 \rho \rho^{\prime}}\right)=4\left(\rho \rho^{\prime}\right)^{-1 / 2}\left(t^{2}-\rho^{2}-{\rho^{\prime 2}}^{2}+2 \rho \rho^{\prime} \cos v\right)^{-1 / 2}
$$

subject to the conditions on (2), appendix.

Put $\cos U=\left(\rho^{2}+\rho^{\prime}-t^{2}\right) / 2 \rho \rho^{\prime}$, then, since in the integration of (7) $\cos U$ varies from 1 to -1 when $t$ varies from $\rho^{\prime}-\rho$ to $\rho^{\prime}+\rho, U$ varies from 0 to $\pi$. But the sum (2), appendix, vanishes when $U<|V|$ [here we write $|V|$ since $V=\phi \mp \phi^{\prime}$ ]. Hence, if (9) is inserted into (7) the integration starts not with $\rho^{\prime}-\rho$ but with $\left(\rho^{2}+{\rho^{\prime 2}}^{2}-2 \rho \rho^{\prime} \cos V\right)^{1 / 2}$ and ends with $\rho+\rho^{\prime}$. If moreover $V>\pi$, the sum vanishes altogether in $0<U<\pi$ and accordingly the integral also.

Since $V=\phi \mp \phi^{\prime}$ we must investigate for which configuration $\phi \mp \phi^{\prime}=\pi$. Let the source point $Q$ be in the upper half plane (because of symmetry this is no restriction), i.e. $0 \leq \phi^{\prime} \leq \pi$ and consider the cases indicated in the Figs. (2) and (3): We form the image $Q^{\prime}$ with respect to the half plane or its extension and draw the straight lines $Q E$ and $Q^{\prime} E$ where $E$ is the edge of the half plane. The domain $0 \leq \phi \leq 2 \pi$ is now subdivided into three regions I, II, III as indicated in the figures.

Region $\mathrm{I}$ is bounded by the upper surface of the half plane and the boundary of geometric reflection.

Region II is bounded by the boundary of geometric reflection and the boundary of geometric shadow.

Region III is bounded by the boundary of geometric shadow and the lower surface of the half plane. 


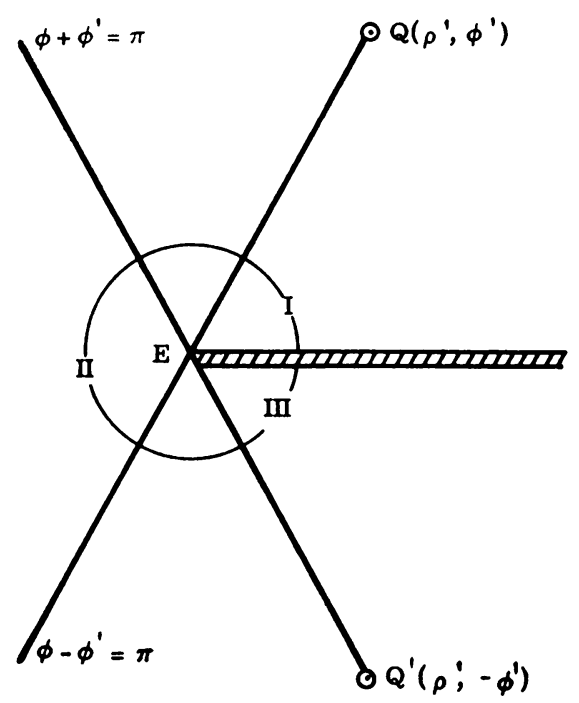

Fig. 2. The half-plane showing the locations of Regions I, II, III for the line source $Q\left(\rho^{\prime}, \phi^{\prime}\right)$ located directly above the half-plane.

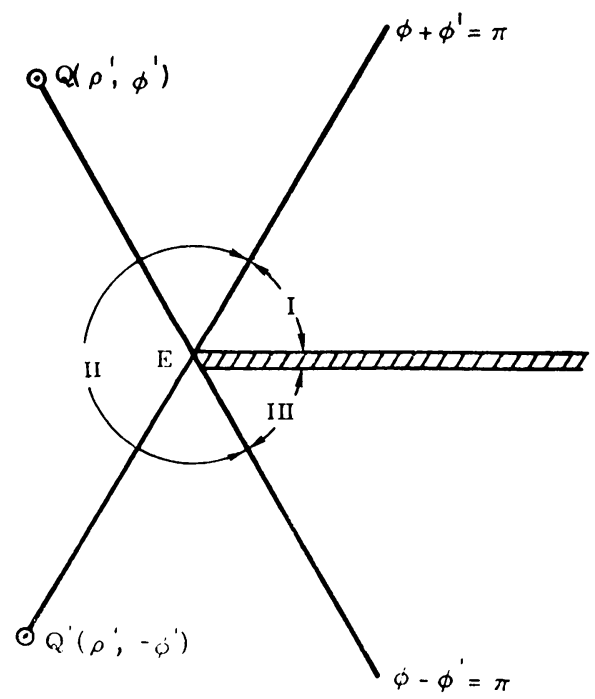

FIG. 3. The half-plane showing the locations of Regions I, II, III for the line source $Q\left(\rho^{\prime}, \phi^{\prime}\right)$ not directly above the half-plane.

Then, for a point of observation $P(\rho, \phi)$ lying in the respective regions:
Region I
$\phi+\phi^{\prime}<\pi$
$\left|\phi-\phi^{\prime}\right|<\pi$,
Region II
$\phi+\phi^{\prime}>\pi$,
$\phi-\phi^{\prime}<\pi$,
Region III
$\phi+\phi^{\prime}>\pi$,
$\phi-\phi^{\prime}>\pi$. 
Hence, by $(9)$

$$
\begin{aligned}
& \int_{\rho^{\prime}-\rho}^{\rho^{\prime}+\rho}\left\{\sum_{0}^{\infty} \epsilon_{n} \cos \frac{n}{2}\left(\phi \mp \phi^{\prime}\right) P_{-1 / 2+n / 2}\left(\frac{\rho^{2}+\rho^{2}-t^{2}}{2 \rho \rho^{\prime}}\right)\right\} e^{-\gamma t} d t \\
& =4\left(\rho \rho^{\prime}\right)^{1 / 2} \int_{\left[\rho^{2}+\rho^{\prime 2}-2 \rho \rho^{\prime} \cos \left(\phi \mp \phi^{\prime}\right)\right]^{1 / 2}}^{\rho^{\prime}+\rho}\left\{t^{2}-\rho^{2}+\rho^{\prime 2}-2 \rho \rho^{\prime} \cos \left(\phi \mp \phi^{\prime}\right)\right\}^{-1 / 2} e^{-\gamma t} d t
\end{aligned}
$$

in the Regions I and II for the upper sign and in Region I for the lower sign, while (10) is zero in Region III for the upper sign and in Regions II and III for the lower sign.

If $K$ and $R^{\prime}$ denote the distance of the point of observation $P$ from the source point $Q$ and its image point $Q^{\prime}$, respectively then:

$$
\begin{aligned}
R & =P Q=\left[\rho^{2}+{\rho^{\prime}}^{2}-2 \rho \rho^{\prime} \cos \left(\phi-\phi^{\prime}\right)\right]^{1 / 2}, \\
R^{\prime} & =P Q^{\prime}=\left[\rho^{2}+{\rho^{\prime}}^{2}-2 \rho \rho^{\prime} \cos \left(\phi+\phi^{\prime}\right)\right]^{1 / 2} .
\end{aligned}
$$

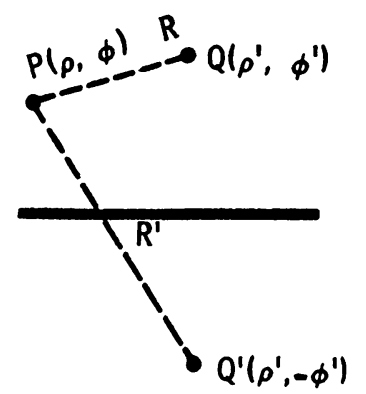

Frg. 4. The half-plane showing the locations of the line source $Q\left(\rho^{\prime}, \phi^{\prime}\right)$ and the image $Q^{\prime}\left(\rho^{\prime},-\phi^{\prime}\right)$ relative to the observer $P(\rho, \phi)$.

Then, from (2), (7), (8) and (10) one obtains for the Green's functions of the two dimensional modified Helmholtz equation: for a point $P$ lying respectively in

Region I

$$
\begin{aligned}
& \left.\begin{array}{l}
G_{1}^{\prime} \\
G_{2}^{\prime}
\end{array}\right\}=-\frac{1}{2 \pi}\left[\int_{R}^{\rho+\rho^{\prime}}\left(t^{2}-R^{2}\right)^{-1 / 2} e^{-\gamma t} d t \mp \int_{R^{\prime}}^{\rho+\rho^{\prime}}\left(t^{2}-R^{\prime 2}\right)^{-1 / 2} e^{-\gamma t} d t\right] \\
& -\frac{1}{4 \pi}\left[\int_{\rho+\rho^{\prime}}^{\infty}\left(t^{2}-R^{2}\right)^{-1 / 2} e^{-\gamma t} d t \mp \int_{\rho+\rho^{\prime}}^{\infty}\left(t^{2}-R^{\prime 2}\right)^{-1 / 2} e^{-\gamma t} d t\right] .
\end{aligned}
$$

Region II

$$
\begin{aligned}
& \left.\begin{array}{l}
G_{1}^{\prime} \\
G_{2}^{\prime}
\end{array}\right\}=-\frac{1}{2 \pi} \int_{R}^{\rho+\rho^{\prime}}\left(t^{2}-R^{2}\right)^{-1 / 2} e^{-\gamma t} d t \\
& -\frac{1}{4 \pi}\left[\int_{\rho+\rho^{\prime}}^{\infty}\left(t^{2}-R^{2}\right)^{-1 / 2} e^{-\gamma \nu} d t \mp \int_{\rho+\rho^{\prime}}^{\infty}\left(t^{2}-R^{\prime 2}\right)^{-1 / 2} e^{-\gamma t} d t\right] .
\end{aligned}
$$

Region III

$$
\left.\begin{array}{l}
G_{1}^{\prime} \\
G_{2}^{\prime}
\end{array}\right\}=-\frac{1}{4 \pi}\left[\int_{\rho+\rho^{\prime}}^{\infty}\left(t^{2}-R^{2}\right)^{-1 / 2} e^{-\gamma t} d t \mp \int_{\rho+\rho^{\prime}}^{\infty}\left(t^{2}-R^{\prime 2}\right)^{-1 / 2} e^{-\gamma t} d t\right] .
$$


This is the desired form (3). Note that the integrand is elementary. We write

$$
\int_{R}^{\rho+\rho^{\prime}}\left(t^{2}-R^{2}\right)^{-1 / 2} e^{-\gamma t} d t=\int_{R}^{\infty}\left(t^{2}-R^{2}\right)^{-1 / 2} e^{-\gamma t} d t-\int_{\rho+\rho^{\prime}}^{\infty}\left(t^{2}-R^{2}\right)^{-1 / 2} e^{-\gamma t} d t
$$

and observe that $[5$, vol. 2, p. 82$]$

$$
\int_{R}^{\infty}\left(t^{2}-R^{2}\right)^{-1 / 2} e^{-\gamma t} d t=K_{0}(\gamma R)
$$

Then $G_{1}^{\prime}$ and $G_{2}^{\prime}$ can be written in the form:

\section{Region I}

$$
\begin{aligned}
& \left.\begin{array}{l}
G_{1}^{\prime} \\
G_{2}^{\prime}
\end{array}\right\}=-\frac{1}{2 \pi} K_{0}(\gamma R) \mp K_{0}\left(\gamma R^{\prime}\right) \\
& +\frac{1}{4 \pi}\left[\int_{\rho+\rho^{\prime}}^{\infty}\left(t^{2}-R^{2}\right)^{-1 / 2} e^{-\gamma t} d t \mp \int_{\rho+\rho^{\prime}}^{\infty}\left(t^{2}-R^{\prime 2}\right)^{-1 / 2} e^{-\gamma t} d t\right]
\end{aligned}
$$

with the physical interpretation that the field consists of two lines sources, one located at $Q$ (the original source) and one located at its image point $Q^{\prime}$, plus an additional "diffraction" term.

Region II

$\left.\begin{array}{l}G_{1}^{\prime} \\ G_{2}^{\prime}\end{array}\right\}=-\frac{1}{2 \pi} K_{0}(\gamma R)+\frac{1}{4 \pi}\left[\int_{\rho+\rho^{\prime}}^{\infty}\left(t^{2}-R^{2}\right)^{-1 / 2} e^{-\gamma t} d t \pm \int_{\rho+\rho^{\prime}}^{\infty}\left(t^{2}-R^{\prime 2}\right)^{-1 / 2} e^{-\gamma t} d t\right]$

where the field consists of a single line source located at $Q$ plus a "diffraction" term.

Region III

$$
\left.\begin{array}{l}
G_{1}^{\prime} \\
G_{2}^{\prime}
\end{array}\right\}=-\frac{1}{4 \pi}\left[\int_{\rho+\rho^{\prime}}^{\infty}\left(t^{2}-R^{2}\right)^{-1 / 2} e^{-\gamma t} d t \mp \int_{\rho+\rho^{\prime}}^{\infty}\left(t^{2}-R^{\prime 2}\right)^{-1 / 2} e^{-\gamma t} d t\right]
$$

where the field consists of a "diffraction" term. Note that the "diffraction" terms are not identical.

Time harmonic case. In order to obtain the corresponding expressions for the time harmonic case (Helmholtz's equation), we have to replace $\gamma$ by $i k$. Since $K_{0}(i z)=$ $-i \pi H_{0}^{(2)}(z) / 2$ it follows from (12a) that

\section{Region I}

$$
\begin{aligned}
& \left.\begin{array}{l}
G_{1}^{\prime} \\
G_{2}^{\prime}
\end{array}\right\}=\frac{i}{4}\left[H_{0}^{(2)}(k R) \mp H_{0}^{(2)}\left(k R^{\prime}\right)\right] \\
& +\frac{1}{4 \pi}\left[\int_{\rho+\rho^{\prime}}^{\infty}\left(t^{2}-R^{2}\right)^{-1 / 2} e^{-i k t} d t \mp \int_{\rho+\rho^{\prime}}^{\infty}\left(t^{2}-R^{\prime 2}\right)^{-1 / 2} e^{-i k t} d t\right] .
\end{aligned}
$$

A similar change occurs in each of the other two regions.

III. Transient solutions. Let us now assume that a "Dirac" pulse is applied to the line source, starting at time $t=0$. The field produced by such a "Dirac" pulse will be labeled $\Phi_{D}(t)$. Then

$$
\Phi_{I}(t)=\mathscr{L}_{\gamma}^{-1}\left\{G_{1,2}^{\prime}\right\}
$$


$\Phi_{D}(t)$ is the inverse Laplace Transform of the Green's functions for the modified Helmholtz equation, with respect to $\gamma$ as inversion parameter [18]. The terms in (12a) to (12c) are expressed as Laplace Transform integrals of the form of (3), whose inversion is the function $F(t)$ itself. Then for a point $P$ lying respectively in the three regions:

Region I

$$
\Phi_{D}(t)=-\frac{1}{2 \pi}\left[\left(t^{2}-R^{2}\right)^{-1 / 2} \mp\left(t^{2}-R^{\prime 2}\right)^{-1 / 2}\right]+\frac{1}{4 \pi}\left[\left(t^{2}-R^{2}\right)^{-1 / 2} \mp\left(t^{2}-R^{\prime 2}\right)^{-1 / 2}\right]
$$

Region II

$$
\Phi_{D}(t)=-\frac{1}{2 \pi}\left(t^{2}-R^{2}\right)^{-1 / 2}+\frac{1}{4 \pi}\left[\left(t^{2}-R^{2}\right)^{-1 / 2} \pm\left(t^{2}-R^{\prime 2}\right)^{-1 / 2}\right]
$$

Region III

$$
\Phi_{D}(t)=-\frac{1}{4 \pi}\left[\left(t^{2}-R^{2}\right)^{-1 / 2} \mp\left(t^{2}-R^{\prime 2}\right)^{-1 / 2}\right]
$$

with the last two terms in (15a) to (15c) equal to zero when $t<\rho+\rho^{\prime}$, while the others are zero for $t<R$ and $t<R^{\prime}$ respectively.

We are now in a position to determine the field $\Phi(t)$ due to an arbitrary time dependent excitation function $g(t)$ applied to the line source at $Q$ [18], under the assumption that $g(t)=0$ for $t<0$.

$$
\Phi(t)=\int_{0}^{\infty} \Phi_{D}(t-\tau) g(\tau) d \tau=\int_{-\infty}^{t} g(t-x) \Phi_{D}(x) d x
$$

where $\Phi_{D}(x)$ is the "Dirac" pulse solution. Then from (15a) to (15c) and (16) we obtain: for a point $P$ lying in

Region I

$$
\begin{aligned}
\Phi(t) & =-\frac{1}{2 \pi} \int_{R}^{t}\left(x^{2}-R^{2}\right)^{-1 / 2} g(t-x) d x \pm \frac{1}{2 \pi} \int_{R^{\prime}}^{t}\left(x^{2}-R^{\prime 2}\right)^{-1 / 2} g(t-x) d x \\
& +\frac{1}{4 \pi}\left[\int_{\rho+\rho^{\prime}}^{t}\left(x^{2}-R^{2}\right)^{-1 / 2} g(t-x) d x \mp \int_{\rho+\rho^{\prime}}^{t}\left(x^{2}-R^{\prime 2}\right)^{-1 / 2} g(t-x) d x\right]
\end{aligned}
$$

Region II

$$
\begin{aligned}
\Phi(t) & =-\frac{1}{2 \pi} \int_{R}^{t}\left(x^{2}-R^{2}\right)^{-1 / 2} g(t-x) d x \\
& +\frac{1}{4 \pi}\left[\int_{\rho+\rho^{\prime}}^{t}\left(x^{2}-R^{2}\right)^{-1 / 2} g(t-x) d x \pm \int_{\rho+\rho^{\prime}}^{t}\left(x^{2}-R^{\prime 2}\right)^{-1 / 2} g(t-x) d x\right],
\end{aligned}
$$

Region III

$\Phi(t)=-\frac{1}{4 \pi}\left[\int_{\rho+\rho^{\prime}}^{t}\left(x^{2}-R^{2}\right)^{-1 / 2} g(t-x) d x \mp \int_{\rho+\rho^{\prime}}^{t}\left(x^{2}-R^{\prime 2}\right)^{-1 / 2} g(t-x) d x\right]$,

with integrals whose upper limit $t$ is smaller than the lower limit $R, R^{\prime}$ and $\rho+\rho^{\prime}$ respectively must be set equal to zero. 
IV. Special cases. As an example for (17a) to (17c) we choose

$$
\begin{aligned}
g(t) & =e^{i \omega t}, & & 0 \leq t \leq T \\
& =0, & & t<0, \quad t>T,
\end{aligned}
$$

namely a time harmonic cylindrical pulse starting at $t=0$ and of duration $T$. The integrals occurring in (17a) to (17c) are then of the form

$$
\int_{c}^{d}\left(x^{2}-a^{2}\right)^{-1 / 2} g(t-x) d x
$$

Since $g(t-x)=0$ for $t-x>T$ two cases must be distinguished:

(a) $t-c>T$,

(b) $t-c<T$.

The lower limit of integration is therefore $t-T$ in case (a) and $c$ in case (b). We then obtain:

\section{Region I}

$$
\begin{aligned}
\Phi(t) e^{-i \omega t}=-\frac{1}{2 \pi} & {\left[\int_{t_{1}}^{t}\left(x^{2}-R^{2}\right)^{-1 / 2} e^{-i \omega x} d x \mp \int_{t_{0}}^{t}\left(x^{2}-{R^{\prime 2}}^{-1 / 2} e^{-i \omega x} d x\right]\right.} \\
& +\frac{1}{4 \pi}\left[\int_{t_{0}}^{t}\left(x^{2}-R^{2}\right)^{-1 / 2} e^{-i \omega x} d x \mp \int_{t_{0}}^{t}\left(x^{2}-{R^{\prime 2}-1 / 2}^{-i \omega x} d x\right]\right.
\end{aligned}
$$

Region II

$$
\begin{aligned}
\Phi(t) e^{-i \omega t}=-\frac{1}{2 \pi} \int_{t_{1}}^{t}\left(x^{2}-R^{2}\right)^{-1 / 2} e^{-i \omega x} d x \\
+\frac{1}{4 \pi}\left[\int_{t_{0}}^{t}\left(x^{2}-R^{2}\right)^{-1 / 2} e^{-i \omega x} d x \pm \int_{t_{0}}^{t}\left(x^{2}-R^{\prime 2}\right)^{-1 / 2} e^{-i \omega x} d x\right]
\end{aligned}
$$

Region III

$$
\Phi(t) e^{-i \omega t}=-\frac{1}{4 \pi}\left[\int_{t_{0}}^{t}\left(x^{2}-R^{2}\right)^{-1 / 2} e^{-i \omega x} d x \mp \int_{t_{0}}^{t}\left(x^{2}-R^{\prime 2}\right)^{-1 / 2} e^{-i \omega x} d x\right] .
$$

The limits are:

$$
\begin{aligned}
& t_{1}=R \text { if } t-R<T \text { and } t_{1}=t-T \text { when } t-R>T, \\
& t_{2}=R^{\prime} \text { if } t-R^{\prime}<T \text { and } t_{2}=t-T \text { when } t-R^{\prime}>T, \\
& t_{3}=\rho+\rho^{\prime} \text { when } t-\left(\rho+\rho^{\prime}\right)<T \text { and } t_{3}=t-T \text { when } t-\left(\rho+\rho^{\prime}\right)>T .
\end{aligned}
$$

The following terms have to be omitted:

The first term in (18a), (18b) when $t<R$, the second term in (18a) when $t<R^{\prime}$ and the last two terms throughout when $t<\rho+\rho^{\prime}$.

We specialize (18a) to (18c) now to the case $\omega=0$, that is, the case of the rectangular pulse of duration $T$ and strength unity. In this case the above integrals become elementary since

$$
\int\left(x^{2}-a^{2}\right)^{-1 / 2} d x=\ln \left[x+\frac{\left(x^{2}-a^{2}\right)^{1 / 2}}{a}\right]
$$


and we obtain

Region I

$$
\begin{aligned}
\Phi(t)=-\frac{1}{2 \pi}\left\{\ln \left[\frac{t+\left(t^{2}-R^{2}\right)^{1 / 2}}{t_{1}+\left(t^{2}-R^{2}\right)^{1 / 2}}\right] \mp \ln \left[\frac{t+\left(t^{2}-R^{\prime 2}\right)^{1 / 2}}{t_{2}+\left(t_{2}^{2}-R^{\prime 2}\right)^{1 / 2}}\right]\right\} \\
+\frac{1}{4 \pi}\left\{\ln \left[\frac{t+\left(t^{2}-R^{2}\right)^{1 / 2}}{t_{3}+\left(t_{3}^{2}-R^{2}\right)^{1 / 2}}\right] \mp \ln \left[\frac{t+\left(t^{2}-R^{\prime 2}\right)^{1 / 2}}{t_{3}+\left(t_{3}^{2}-R^{\prime 2}\right)^{1 / 2}}\right]\right\}
\end{aligned}
$$

Region II

$$
\begin{aligned}
\Phi(t)=-\frac{1}{2 \pi} \ln \left[\frac{t+\left(t^{2}-R^{2}\right)^{1 / 2}}{t_{1}+\left(t_{1}^{2}-R^{2}\right)^{1 / 2}}\right] \\
\quad+\frac{1}{4 \pi}\left\{\ln \left[\frac{t+\left(t^{2}-R^{2}\right)^{1 / 2}}{t_{3}+\left(t_{3}^{2}-R^{2}\right)^{1 / 2}}\right] \pm \ln \left[\frac{t+\left(t^{2}-R^{\prime 2}\right)^{1 / 2}}{t_{3}+\left(t_{3}^{2}-R^{\prime 2}\right)^{1 / 2}}\right]\right\}
\end{aligned}
$$

Region III

$$
\Phi(t)=-\frac{1}{4 \pi}\left\{\ln \left[\frac{t+\left(t^{2}-R^{2}\right)^{1 / 2}}{t_{3}+\left(t_{3}^{2}-R^{2}\right)^{1 / 2}}\right] \mp \ln \left[\frac{t+\left(t^{2}-R^{\prime 2}\right)^{1 / 2}}{t_{3}+\left(t_{3}^{2}-R^{\prime 2}\right)^{1 / 2}}\right]\right\}
$$

with $t_{1}, t_{2}, t_{3}$ as given before. Certain terms have to be replaced by zero under the following conditions:

The first term in (19a) and (19b) when $t<R$, the second term in (19b) when $t<R^{\prime}$, and the last two terms throughout when $t<\rho+\rho^{\prime}$.

We conclude with the case $T=\infty$, i.e., the incident cylindrical pulse is a time harmonic one, starting at $t=0$. Here only case (b) applies, with the limits $t_{1}, t_{2}, t_{3}$ in (18a) to (18c) being: $t_{1}=R, t_{2}=R^{\prime}, t_{3}=\rho+\rho^{\prime}$. Of particular interest is the case $t>\rho+\rho^{\prime}$, i.e., none of the terms in (18a) to (18c) vanish. We then write

$$
\begin{array}{r}
\int_{R}^{t}\left(x^{2}-R^{2}\right)^{-1 / 2} e^{-i \omega x} d x=\int_{R}^{\infty}\left(x^{2}-R^{2}\right)^{-1 / 2} e^{-i \omega x} d x-\int_{t}^{\infty}\left(x^{2}-R^{2}\right)^{-1 / 2} e^{-i \omega x} d x \\
=-\frac{1}{2} i \pi H_{0}^{(2)}(\omega R)-\int_{t}^{\infty}\left(x^{2}-R^{2}\right)^{-1 / 2} e^{-i \omega x} d x,
\end{array}
$$

using the well-known expression for the second Hankel function [5, Vol. 2, p. 21]. A similar expression is used for the term involving $R^{\prime}$. Similarly

$$
\int_{t_{0}}^{t}\left(x^{2}-R^{2}\right)^{-1 / 2} e^{-i \omega x} d x=\int_{\rho+\rho^{\prime}}^{\infty}\left(x^{2}-R^{2}\right)^{-1 / 2} e^{-i \omega x} d x-\int_{t}^{\infty}\left(x^{2}-R^{2}\right)^{-1 / 2} e^{-i \omega x} d x
$$

We insert these results into (18a) to (18c) and group time independent terms together and time dependent terms together. The field $\Phi(t)$ then appears in the form:

$$
\Phi(t) e^{-i \omega t}=G_{1,2}+Y_{T}(t) .
$$

Here $G_{1}$ and $G_{2}$ represent the Green's functions for the time harmonic case (13). $Y_{T}(t)$ represents the transient field, that is, the deviation of the field produced by a time harmonic excitation starting at $t=0$ from the quasi-stationary case of the time harmonic excitation starting at $t=-\infty$ (provided $t>\rho+\rho^{\prime}$ ). We find for the transient field

$$
Y_{T}(t)=\frac{1}{4 \pi}\left[\int_{t}^{\infty} e^{-i \omega x}\left(x^{2}-R^{2}\right)^{-1 / 2} d x \mp \int_{t}^{\infty} e^{-i \omega x}\left(x^{2}-R^{\prime 2}\right)^{-1 / 2} d x\right]
$$


for all three Regions I, II, III. Obviously (22) tends to zero as $t \rightarrow \infty$, i.e. the transient field becomes less and less significant with increasing time.

\section{Appendix}

Some integral and sum formulae. Summation of series involving Legendre functions

$$
\begin{gathered}
\sum_{n=0}^{\infty} \epsilon_{n} P_{-1 / 2+n \pi / \alpha}(\cos u) \cos \left(\frac{n \pi v}{\alpha}\right)=2^{1 / 2} \pi^{-1} \alpha(\cos v-\cos u)^{-1 / 2}, \quad-u<v<u \\
=0, \quad u<v<2 \alpha-u, \\
0<u<\pi, \quad\left(\epsilon_{n} \text { is Neumann's number, } \epsilon_{0}=1, \epsilon_{n}=2, n \geq 1\right) .
\end{gathered}
$$

The formula (1) is a Fourier series with respect to $v$ with period $v=2 \alpha$. To prove (1) we expand the function

$$
\begin{aligned}
f(v) & =(\cos v-\cos u)^{-1 / 2}, \quad-u<v<u \\
& =0, \quad \text { otherwise, }
\end{aligned}
$$

into a Fourier series of period $2 \alpha$. Since this is an even function of $v$, we have, by Fourier's theorem:

with

$$
f(v)=\sum_{n=0}^{\infty} A_{n} \cos \left(\frac{n \pi v}{\alpha}\right)
$$

$$
A_{n}=\epsilon_{n}(2 \alpha)^{-1} \int_{-\alpha}^{\alpha} f(v) \cos \left(\frac{n \pi v}{\alpha}\right) d v
$$

therefore for $\alpha \geq u$

$$
A_{n}=\epsilon_{n}(2 \alpha)^{-1} \int_{-u}^{u}(\cos v-\cos u)^{-1 / 2} \cos \left(\frac{n \pi v}{\alpha}\right) d v .
$$

This integral is known [5, Vol. 1, p. 159, formula 27].

$$
A_{n}=\epsilon_{n} \alpha^{-1} 2^{-1 / 2} \pi P_{-1 / 2+n \pi / \alpha}(\cos u) \text {. }
$$

This proves (1).

The special case $\alpha=2 \pi$ yields

$$
\begin{aligned}
\sum_{n=0}^{\infty} \epsilon_{n} P_{n / 2-1 / 2}(\cos u) \cos \left(\frac{n v}{2}\right) & =2^{3 / 2}(\cos v-\cos u)^{-1 / 2}, \quad-u<v<u \\
& =0, \quad u<v<4 \pi-u
\end{aligned}
$$

$0<u<\pi$.

The sum for (8) is known [5, Vol. 1, p. 166]

$$
\sum_{n=0}^{\infty}(-1)^{n} \epsilon_{n} Q_{n-1 / 2}(z) \cos (n v)=2^{-1 / 2} \pi(z+\cos v)^{-1 / 2}, \quad z \geq 1 .
$$

Representation of the product of two modified Bessel functions as a Laplace transform. We prove the formulae:

$$
\begin{aligned}
I_{\nu}(b s) K_{v}(a s)=\frac{1}{2}(a b)^{-1 / 2}\left\{\int_{a-b}^{a+b}\right. & P_{\nu-1 / 2}\left(\frac{a^{2}+b^{2}-t^{2}}{2 a b}\right) e^{-s t} d t \\
& \left.+2 \pi^{-1} \cos (\pi \nu) \int_{a+b}^{\infty} Q_{\nu-1 / 2}\left(\frac{t^{2}-a^{2}-b^{2}}{2 a b}\right) e^{-\imath t} d t\right\}
\end{aligned}
$$




$$
K_{\nu}(a s) K_{\nu}(b s)=\frac{1}{2} \pi(a b)^{-1 / 2} \int_{a+b}^{\infty} \mathcal{P}_{\nu-1 / 2}\left(\frac{t^{2}-a^{2}-b^{2}}{2 a b}\right) e^{-s t} d t .
$$

If (4) is established, (5) follows by the relation

$$
K_{\nu}(x)=\pi[2 \sin (\pi \nu)]^{-1}\left[I_{-\nu}(x)-I_{\nu}(x)\right]
$$

In order to prove (4) we start with the well-known formula [5, Vol. 2, p. 96]

$$
I_{\nu}(b s) K_{\nu}(a s)=\int_{0}^{\infty} v\left(v^{2}+s^{2}\right)^{-1} J_{\nu}(a v) J_{\nu}(b v) d v
$$

$a \geq b, \quad \operatorname{Re} \nu>-1$.

In the integrand above we substitute

$$
v\left(v^{2}+s^{2}\right)^{-1}=\int_{0}^{\infty} e^{-s t} \sin (v t) d t
$$

and obtain, upon interchanging the order of integration

$$
I_{\nu}(b s) K_{\nu}(a s)=\int_{0}^{\infty} e^{-s t}\left[\int_{0}^{\infty} J_{\nu}(a v) J_{\nu}(b v) \sin (v t) d v\right] d t
$$

The inner integral is known [19, p. 169]

$$
\begin{aligned}
\int_{0}^{\infty} J_{\nu}(a v) J_{\nu}(b v) \sin (v t) d v & =0, \quad 0<t<a-b, \\
& =\frac{1}{2}(a b)^{-1 / 2} P_{\nu-1 / 2}\left(\frac{a^{2}+b^{2}-t^{2}}{2 a b}\right), \quad a-b<t<a+b, \\
& =\pi^{-1}(a b)^{-1 / 2} \cos (\pi v) Q_{\nu-1 / 2}\left(\frac{t^{2}-a^{2}-b^{2}}{2 a b}\right), \quad t \geq a .
\end{aligned}
$$

This proves (4).

\section{List of notations.}

$$
\begin{aligned}
J_{\nu}(z) & =\sum_{n=0}^{\infty} \frac{(-1)^{n}(z / 2)^{\nu+2 n}}{n !(\nu+n+1)}, \\
Y_{\nu}(z) & =(\sin \pi \nu)^{-1}\left[J_{\nu}(z) \cos (\pi \nu)-J_{-\nu}(z)\right], \\
H_{\nu}^{(2)}(z) & =J_{\nu}(z)-i Y_{\nu}(z),
\end{aligned}
$$

Bessel function, Neumann function and Hankel function, respectively.

$$
\begin{aligned}
& I_{\nu}(z)=e^{-i \pi \nu / 2} J_{\nu}\left(z e^{i \pi / 2}\right), \\
& K_{\nu}(z)=\pi[2 \sin (\pi \nu)]^{-1}\left[I_{-\nu}(z)-I_{\nu}(z)\right]
\end{aligned}
$$

modified Bessel function and modified Hankel function, respectively.

$$
K_{\nu}\left(z e^{i \pi / 2}\right)=-\frac{1}{2} i \pi e^{-i \pi / 2} H_{\nu}^{(2)}(z)
$$

(see [5, Vol. 2, Ch. 7]).

$P_{v}(z)$ Legendre function of the first kind, $-1<x<1$.

$\mathcal{P}_{\nu}(z)$ Legendre function of the first kind, $z$ not on the real axis between 1 and $-\infty$.

$Q_{\nu}(z)$ Legendre function of the second kind, $z$ not on the real axis between 1 and $-\infty$. (See [5, Vol. 1, Ch. 3].) 
Acknowledgment. The writer wishes to thank Professor F. Oberhettinger for suggesting this research and for his advice and guidance during the investigation.

\section{REFERENCES}

1. L. Cagniard, Reflection and refraction of progressive seismic waves, McGraw-Hill, New York, 1962

2. P. C. Clemmow, A note on the diffraction of a cylindrical wave by a perfectly conducting half plane, Quart. J. Mech. Appl. Math. 3, 377 (1950)

3. A. T. DeHoop, A modification of Cagniard's method for solving seismic pulse problems, Appl. Sci. Res. (B) 8, 349-356 (1960)

4. C. Hewitt Dix, The method of Cagniard in seismic pulse problems, Geophysics 19, 722-738 (1954)

5. A. Erdelyi, Higher transcendental functions, Vol. 1, 2, McGraw-Hill, New York, 1953

6. F. G. Friedlander, The diffraction of sound pulses, Proc. Roy. Soc., London (A) 186, 322 (1946)

7. F. G. Friedlander, On the half plane diffraction problem, Quart. J. Mech. Appl. Math. 4, 344 (1951)

8. F. G. Friedlander, Sound pulses, Cambridge University Press, Cambridge, 1958

9. H. G. Garnir, Fonctions de Green des opérateurs $\Delta-k^{2},(k>0), \Delta-\left(1 / c^{2}\right)\left(\partial^{2} / \partial t^{2}\right),(c>0), \Delta-$ $(1 / k)(\partial / \partial t),(k>0)$ pour les problémes de Dirichlet et de Neumann posés dans un segment, une bande ou une dalle, Soc. Roy. des Sci. Liege 22, 29-46 (1953)

10. R. C. Gast, Half plane diffraction with line source excitation, Technical Report No. 24 Carnegie Institute of Technology, Pittsburgh, 1956

11. R. F. Harrington, Current element near the edge of a conducting half plane, J. Appl. Phys. 24, 547 (1953)

12. I. Kay, The diffraction of an arbitrary pulse by a wedge, Comm. Pure and Appl. Math. 6, 419 (1953)

13. J. B. Keller and A. Blank, Diffraction and reflection of pulses by wedges and corners, Comm. Pure and Appl. Math. 4, 75 (1951)

14. M. J. Kontorovich and N. N. Lebedev, On a method of solution of some problems in diffraction theory, J. Phys. Moscow 1, 229 (1939)

15. J. W. Miles, On the diffraction of an acoustic pulse by a wedge, Proc. Roy. Soc., London (A) 212, $543(1952)$

16. J. W. Miles, On the diffraction of an electromagnetic pulse by a wedge, Proc. Roy. Soc., London (A) 212, 547 (1952)

17. F. Oberhettinger, Diffraction of waves by a wedge, Comm. Pure Appl. Math. 7, 551 (1953)

18. F. Oberhettinger, On the diffraction of waves and pulses by wedges and corners, J. Res. Nat. Bureau of Standards 61, 343 (1958)

19. F. Oberhettinger, Tabelen zur Fourier Transformation, Springer, Berlin, 1957, p. 169

20. A. Sommerfeld, Partial differential equations in physics, Academic Press, New York, 1964

21. R. D. Turner, The diffraction of a cylindrical pulse by a half plane, Quart. Appl. Math. 14, 63 (1956)

22. J. Wait, Diffraction of a spherical wave pulse by a half plane screen, Canad. J. Phys. 35, 693 (1957)

23. C. P. Wells and A. Leitner, A Lebedev transform and the "baffle" problem, Quart. Appl. Math. 15, $430(1958)$ 